03

\title{
Об устойчивости поверхности короткой заряженной струи, движущейся относительно внешней материальной среды
}

\author{
(ㄱ Н.А. Петрушов, А.И. Григорьев, С.О. Ширяева
}

Ярославский государственный университет им. П.Г. Демидова, 150000 Ярославль, Россия

e-mail: grig@uniyar.ac.ru

(Поступило в Редакцию 1 марта 2017 г.)

Рассмщтрена задача об устойчивости капиллярных волн на поверхности заряженной струи идеальной несжимаемой электропроводной жидкости, движущейся относительно материальной диэлектрической среды. На поверхности раздела сред имеется тангенциальный разрыв поля скоростей. Сравниваются решения задачи в двух идеализированных моделях: когда струя имеет конечную и бесконечную длину. Показано, что инкременты неустойчивости и волновые числа наиболее неустойчивых волн, рассчитанные в обеих моделях, линейно связаны, а коэффициентом пропорциональности выступает скорость движения струи.

DOI: $10.21883 /$ JTF.2017.12.45200.2224

\section{Введение}

При аналитическом исследовании устойчивости и дробления на капли цилиндрической жидкой струи, движущейся относительно среды, можно исходить из двух посылок: принимая струю бесконечно длинной (идеальная модель с точки зрения теоретика), как предложил Рэлей [1-5] или, считая ее конечной, вытекающей из сопла и дробящейся на некотором расстоянии от среза сопла (модель, близкая к реальной ситуации), как предложили спустя почти век в [7-11].

Исторически первое аналитическое исследование устойчивости цилиндрической струи идеальной жидкости выполнено для бесконечной струи в конце позапрошлого века Рэлеем [1]. В последующих теоретических работах Рэлея [2], Бассета [3], Вебера [4] на модели бесконечной струи было исследовано влияние на устойчивость струи: вязкости жидкости, наличия внешней материальной среды и индуцированного заряда. Но первый заметный вклад в изучении влияния заряда на устойчивость струи сделал Тейлор в рамках той же модели лишь в конце шестидесятых годов прошлого века [5].

Справедливости ради следует отметить, что, хотя теория устойчивости струи с начала исследований в конце позапрошлого века и начале прошлого делалась на модели бесконечной струи, эксперименты, на которые опирались теоретики, выполнялись со струями конечной длины.

Рэлей для бесконечной струи идеальной жидкости в вакууме вывел дисперсионное уравнение в виде

$$
\omega^{2}=\frac{\sigma}{\rho R^{3}} \frac{k R \cdot J_{m}^{\prime}(k R)}{J_{m}(k R)} \cdot\left(m^{2}+k^{2} R^{2}-1\right),
$$

где $\omega-$ частота, в общем случае комплексная; $k-$ волновое число, вещественное; $\sigma$ и $\rho-$ коэффициент поверхностного натяжения и плотность жидкости струи; $R$ - ее радиус; $m$ - азимутальное число;
$J_{m}$ - функция Бесселя. Появление мнимой части $\gamma$ у частоты соответствует экспоненциальному возрастанию во времени с показателем амплитуды волны, т.е. к проявлению неустойчивости. Показатель $\gamma$ назвали инкрементом неустойчивости. Рэлей нашел, что максимальное значение инкремент неустойчивости достигает при $K^{2} R^{2} \approx 0.4858$, что соответствует длине волны $\lambda \approx 9.016 \cdot R$. Волны с указанной длиной определяют закономерности дробления струи на отдельные капли.

Согласно теории Рэлея, появление мнимой части у частоты $\omega$ приводит к неустойчивости струи на протяжении всей ее бесконечной длины. Саму неустойчивость бесконечной струи по отношению к капиллярным волнам назвали временной неустойчивостью.

В реальности любое возмущение поверхности струи весьма мало вблизи сопла (из которого вытекает) и увеличивается по мере удаления от него. Для описания реальных струй (лет через 80 после появления первой работы Рэлея по струям) была развита теоретическая модель пространственной неустойчивости [7-12]. Для струи конечной длины частота $\omega$ принимается вещественной, а волновое число $k$ в общем случае комплексным, саму же неустойчивость называли пространственной неустойчивостью.

При той же формальной постановке исследование пространственной неустойчивости более сложно и громоздко, чем исследование временной неустойчивости изза того, что частота и волновое число входят в дисперсионное уравнение по-разному. Когда мы пытаемся его разрешить относительно волнового числа, то сталкиваемся с необходимостью разрешения трансцендентного уравнения. В то время как при исследовании временной неустойчивости дисперсионное уравнение относительно частоты является степенным уравнением, решение которого даже в аналитическом виде не представляет сложности.

Как результат, подход Рэлея оказался более плодотворным и нашел большое количество последователей 
(см., например, обзоры [12-17] и указанную в них литературу). В частности, в авторском коллективе настоящей работы с использованием модели струи бесконечной длины было проведено исследование капиллярного волнового движения на поверхности заряженной струи, движущейся относительно внешней материальной среды [18-19].

В связи со сказанным, представляется целесообразным провести исследование пространственной неустойчивости капиллярных волн в постановке, аналогичной использованной в $[18,19]$, и установить сходство и различие двух описываемых подходов.

\section{Постановка задачи}

В связи со сказанным выше проведем исследование пространственной неустойчивости капиллярных волн на поверхности заряженной струи радиусом $R$ и поверхностной плотностью заряда $\chi$ несжимаемой идеальной идеально проводящей жидкости плотностью $\rho_{1}$, движущейся со скоростью $\mathbf{U}_{0}$ относительно внешней среды, которую будем моделировать несжимаемой идеальной диэлектрической жидкостью с плотностью $\rho_{2}$. Коэффициент межфазного натяжения на границе раздела сред равен $\sigma$.

Задача решается в безразмерных переменных, в которых принято $R=\sigma=\rho_{1}=1$, в цилиндрической системе координат, ось $O Z$ которой совпадает с осью симметрии невомущенной струи. Начало системы координат свяжем с торцом сопла, из которого вытекает струя.

Математическая постановка задачи имеет вид

$$
\begin{aligned}
& \partial_{t} \mathbf{u}_{1}(\mathbf{r}, t)+\left(\mathbf{u}_{1}, \nabla\right) \mathbf{u}_{1}(\mathbf{r}, t)=-\nabla p_{1}(\mathbf{r}, t) ; \\
& \partial_{t} \mathbf{u}_{2}(\mathbf{r}, t)+\left(\mathbf{u}_{2}, \nabla\right) \mathbf{u}_{2}(\mathbf{r}, t)=-\frac{1}{\rho} \nabla p_{2}(\mathbf{r}, t) ; \\
& \operatorname{divu}_{1}(\mathbf{r}, t)=0 ; \quad \operatorname{divu}_{2}(\mathbf{r}, t)=0 ; \quad \operatorname{div} \mathbf{E}(\mathbf{r}, t)=0 ; \\
& r \rightarrow \infty: \quad \mathbf{E} \rightarrow 0 ; \quad \mathbf{u}_{2} \rightarrow 0 ; \\
& r \rightarrow 0: \quad \mathbf{u}_{1} \rightarrow \mathbf{U}_{0} \\
& r=1+\xi(\varphi, z, t): \quad \frac{d F}{d t}=0, \quad F \equiv r-1-\xi(\varphi, z, t) \text {; } \\
& \left(\mathbf{n}, \mathbf{u}_{1}\right)=\left(\mathbf{n}, \mathbf{u}_{2}\right)=u_{n}(\mathbf{r}, t) ; \quad \Phi(\mathbf{r}, t)=\Phi_{s}(t) ; \\
& p_{1}(\mathbf{r}, t)-\left(p_{2}(\mathbf{r}, t)\right)+p_{E}(\mathbf{r}, t)-p_{\sigma}(\mathbf{r}, t)=0 \text {; } \\
& p_{E}(\mathbf{r}, t) \equiv \frac{E^{2}(\mathbf{r}, t)}{4 \pi} ; \quad p_{\sigma}(\mathbf{r}, t) \equiv \operatorname{div} \mathbf{n}(\mathbf{r}, t) ; \\
& \mathbf{n}(\mathbf{r}, t) \equiv \frac{\nabla F}{|\nabla F|} ; \quad \rho \equiv \frac{\rho_{2}}{\rho_{1}},
\end{aligned}
$$

где $\xi(\varphi, z, t)$ - возмущение на поверхности струи, $|\xi|=R ; \chi=E_{n} / 4 \pi$ - поверхностная плотность заряда; $\Phi(\mathbf{r}, t)$ - электростатический потенциал; $\mathbf{u}_{1}(\mathbf{r}, t)$ и $\mathbf{u}_{2}(\mathbf{r}, t)$ - поля скоростей в струе и внешней среде соответственно; $p_{1}(\mathbf{r}), \mathbf{t}$ и $p_{2}(\mathbf{r}, t)$ - гидродинамические давления в струе и внешней среде соответственно; $p_{E}(\mathbf{r}, t)$ и $p_{\sigma}(\mathbf{r}, t)$ - давление электрических и капиллярных сил соответственно; $\mathbf{n}(\mathbf{r}, t)$ - орт внешней нормали к поверхности струи.

\section{Методика решения}

Выразим напряженность электростатического поля через электростатический потенциал $\Phi(\mathbf{r}, t)$, и, используя модель потенциального течения жидкости, перейдем от скоростей к гидродинамическим потенциалам $\psi_{1,2}(\mathbf{r}, t)$ :

$$
\begin{gathered}
\mathbf{E}(\mathbf{r}, t)=-\nabla \Phi(\mathbf{r}, t) ; \quad \mathbf{u}_{1}(\mathbf{r}, t)=\mathbf{U}_{0}+\nabla \psi_{1}(\mathbf{r}, t), \\
\mathbf{u}_{2}(\mathbf{r}, t)=\nabla \psi_{2}(\mathbf{r}, t) .
\end{gathered}
$$

Решение задачи ищется асимптотическим методом путем разложения неизвестных функций по малому параметру, в качестве которого выступает отношение максимума модуля амплитуд волн к радиусу струи $(\varepsilon \equiv \max |\xi| / R)$ с точностью до пренебрежения слагаемыми пропорциональными квадрату малого параметра:

$$
\begin{gathered}
\psi_{1}(\mathbf{r}, t)=\varepsilon \psi_{1}^{(1)}(\mathbf{r}, t)+O\left(\varepsilon^{2}\right) ; \\
\psi_{2}(\mathbf{r}, t)=\varepsilon \psi_{2}^{(1)}(\mathbf{r}, t)+O\left(\varepsilon^{2}\right) ; \\
\Phi(\mathbf{r}, t)=\Phi^{(0)}(\mathbf{r})+\varepsilon \Phi^{(1)}(\mathbf{r}, t)+O\left(\varepsilon^{2}\right) \\
\xi(\mathbf{r}, t)=\varepsilon \xi^{(1)}(\mathbf{r}, t)+O\left(\varepsilon^{2}\right) ; \\
p_{i}(\mathbf{r}, t)=p_{i}^{0}+\varepsilon p_{i}^{(1)}(\mathbf{r}, t)+O\left(\varepsilon^{2}\right) .
\end{gathered}
$$

На порядок малости указывает верхний индекс, стоящий в скобках

Подставляя указанные разложения в задачу (2), разобьем ее по порядкам малости.

Задача нулевого порядка малости описывает стационарное состояние и имеет вид

$$
\begin{gathered}
\mathbf{u}_{1}=\mathbf{U}_{0} ; \quad \mathbf{u}_{2}=0 ; \quad p_{1}^{(0)}=-\frac{U_{0}^{2}}{2}+C_{1} ; \\
p_{2}^{(0)}=\rho C_{2} ; \quad \Delta \Phi_{0}(r)=0 ; \\
r \rightarrow \infty: \quad \Phi_{0}(r) \rightarrow 0 ; \quad r \rightarrow 1: \quad \Phi_{0}(r)=\Phi_{x}^{(0)} ; \\
-\left.\frac{1}{4 \pi} \int_{0}^{2 \pi} \int_{z_{0}}^{z_{0}+\lambda} \frac{\partial \Phi_{0}(r)}{\partial r}\right|_{r=1} d \varphi d z=2 \pi \chi \lambda ; \\
p_{1}^{(0)}-p_{2}^{(0)}+\frac{1}{8 \pi}\left(\nabla \Phi_{0}(r)\right)^{2}-1=0,
\end{gathered}
$$

где $\lambda$ - длина капиллярной волны на струе.

Ее решение легко выписывается

$$
\begin{gathered}
\mathbf{u}_{1}(\mathbf{r}, t)=\mathbf{U}_{0} ; \quad \mathbf{U}_{2}(\mathbf{r}, t)=0 ; \quad \Phi_{0}(r)=-4 \pi \chi \ln r \\
p_{2}^{(0)}=p_{0} ; \quad p_{1}^{(0)}=p_{0}-2 \pi \chi^{2}+1 .
\end{gathered}
$$

Здесь $p_{j}^{(0)}$, где $(\mathrm{j}=1,2)$, и $\Phi_{0}(r)-$ гидродинамические давления в обеих средах и электростатический потенциал в стационарном состоянии; $p_{0}$ - константа, равная гидростатическому давлению во внешней среде. 
Задача первого порядка малости запишется в виде

$$
\begin{aligned}
& \Delta \psi_{1}^{(1)}(\mathbf{r}, t)=0 ; \quad \Delta \psi_{2}^{(1)}(\mathbf{r}, t)=0 ; \quad \Delta \Phi^{(1)}(\mathbf{r}, t)=0 ; \\
& r \rightarrow 0: \quad \psi_{1}^{(1)}(\mathbf{r}, t) \rightarrow 0 \\
& r \rightarrow \infty: \quad \psi_{2}^{(1)}(\mathbf{r}, t) \rightarrow 0 ; \quad \Phi^{(1)}(\mathbf{r}, t) \rightarrow 0 ; \\
& r=1: \quad \frac{\partial \psi_{1}^{(1)}(\mathbf{r}, t)}{\partial r}=\frac{\partial \xi^{(1)}(\varphi, z, t)}{\partial t}+U_{0} \frac{\partial \xi^{(1)}(\varphi, z, t)}{\partial z} ; \\
& \frac{\partial \psi_{2}^{(1)}(\mathbf{r}, t)}{\partial r}=\frac{\partial \xi^{(1)}(\varphi, z, t)}{\partial t} \\
& \frac{\partial \Phi^{(0)}(r)}{\partial r} \xi^{(1)}(\varphi, z, t)+\Phi^{(1)}(\mathbf{r}, t)=0 ; \\
& -\frac{\partial \psi_{1}^{(1)}(\mathbf{r}, t)}{\partial t}+\rho \frac{\partial \psi_{2}^{(1)}(\mathbf{r}, t)}{\partial t}-\left(\mathbf{U}_{0}, \nabla \psi_{1}^{(1)}(\mathbf{r}, t)\right) \\
& +\frac{1}{4 \pi}\left(\nabla \Phi^{(0)}(r), \nabla \Phi_{1}^{(1)}(\mathbf{r}, t)\right)+\frac{1}{8 \pi} \frac{\partial\left(\nabla \Phi^{(0)}(r)\right)^{2}}{\partial r} \\
& \times \xi^{(1)}(\varphi, z, t)+\xi^{(1)}(\varphi, z, t)+\Delta_{S} \xi^{(1)}(\varphi, z, t)=0 ; \\
& \int_{0}^{2 \pi} \int_{z_{0}}^{z_{0}+\lambda} \xi^{(1)}(\varphi, z, t) d \varphi d z=0 \\
& \int_{0}^{2 \pi} \int_{z_{0}}^{z_{0}+\lambda}\left(\frac{\partial \Phi^{(0)}(r)}{\partial r} \xi^{(1)}(\varphi, z, t)+\frac{\partial^{2} \Phi^{(0)}(r)}{\partial r^{2}} \xi^{(1)}(\varphi, z, t)\right. \\
& \left.+\frac{\partial \Phi^{(1)}(\mathbf{r}, t)}{\partial r}\right) d \varphi d z=0 \\
& \Delta_{S} \equiv \frac{1}{r^{2}} \frac{\partial^{2}}{\partial \varphi^{2}}+\frac{\partial^{2}}{\partial z^{2}} .
\end{aligned}
$$

Решение задачи (3) будем искать в виде элементарных бегущих волн

$$
\begin{aligned}
& \xi^{(1)}(\varphi, z, t)=\alpha_{1} \exp (i k z+i m \varphi)+\alpha_{2} \exp (i k z-i m \varphi) \\
& +\alpha_{3} \exp (-i k z+i m \varphi)+\alpha_{4} \exp (-i k z-i m \varphi)+O\left(\varepsilon^{2}\right) \\
& \Phi^{(1)}(\mathbf{r}, t)=K_{m}(k r)\left[a_{1} \exp (i k z+i m \varphi)+a_{2}(i k z-i m \varphi)\right. \\
& \left.+a_{3} \exp (-i k z+i m \varphi)+a_{4} \exp (-i k z-i m \varphi)\right]+O\left(\varepsilon^{2}\right) \\
& \psi_{1}^{(1)}(\mathbf{r}, t)=I_{m}(k r)\left[b_{1} \exp (i k z+i m \varphi)+b_{2} \exp (i k z-i m \varphi)\right. \\
& \left.+b_{3} \exp (-i k z+i m \varphi)+b_{4} \exp (-i k z-i m \varphi)\right]+O\left(\varepsilon^{2}\right) \\
& \psi_{2}^{(1)}(\mathbf{r}, t)=K_{m}(k r)\left[c_{1} \exp (i k z+i m \varphi)+c_{2}(i k z-i m \varphi)\right. \\
& \left.+c_{3} \exp (-i k z+i m \varphi)+c_{4} \exp (-i k z-i m \varphi)\right]+O\left(\varepsilon^{2}\right)
\end{aligned}
$$

где $I_{m}(k r)$ и $K_{m}(k r)$ - модифицированные функции Бесселя первого и второго рода, порядка $m$ [20], $m$ - азимутальный параметр, который может принимать целочисленные значения. Для $m=1,2,3$ профили поверхности струй и закономерности реализации их неустойчивости по отношению к заряду, приходящемуся на единицу длины струи, известны из экспериментов (см., например, [21-23]).

Кинематическое граничное условие и условие эквипотенциальности поверхности струи позволяют выразить неизвестные амплитуды $a_{j}(t), b_{j}(t)$ и $c_{j}(t)$ в проектах решений для гидродинамических и электростатического потенциалов через амплитуду $\alpha_{j}(t)$ волн на границе раздела сред

$$
\begin{gathered}
a_{j}=\alpha_{j}(t) \frac{4 \pi \chi}{K_{m}(k)}, \quad b_{j}(t)=\frac{\alpha_{j}^{\prime}(t) \mp i k U_{0} \alpha_{j}(t)}{k I_{m}^{\prime}(k)}, \\
c_{j}(t)=\frac{\alpha_{j}^{\prime}(t)}{k K_{m}^{\prime}(k)} .
\end{gathered}
$$

Подставляя проекты решений (4) с учетом полученных соотношений в динамическое граничное условие, получим дифференциальное уравнение относительно неизвестной амплитуды $\alpha(t)$ :

$$
\begin{gathered}
\beta_{m} \alpha_{j}^{\prime \prime}(t) \mp 2 i \delta_{m} \alpha_{j}^{\prime}(t)+\kappa_{m} \alpha_{j}(t)=0 ; \\
\beta_{m}(k)=\equiv \rho h_{m}^{-1}-g_{m}^{-1} \equiv \frac{\rho g_{m}-h_{m}}{g_{m} h_{m}} ; \\
\delta_{m}\left(k, U_{0}\right) \equiv k U_{0} g_{m}^{-1}=k \sqrt{\frac{\mathrm{We}}{\rho} g_{m}^{-1}} ; \\
\kappa_{m}\left(k, \chi, U_{0}\right) \equiv 1-m^{2}-k^{2}-4 \pi \chi^{2}\left(1+h_{m}\right)+k^{2} U_{-}^{2} g_{m}^{-1} \\
=1-m^{2} k^{2}-w\left(1+h_{m}\right)+k^{2} \frac{W e}{\rho} g_{m}^{-1} ; \\
w=4 \pi \chi^{2} ; \quad \mathrm{We} \equiv \rho U_{0}^{2} ; \\
h_{m}(k) \equiv \frac{k K_{m}^{\prime}(k)}{K_{m}(k)} \equiv m-\frac{k K_{m+1}(k)}{K_{m}(k)} ; \\
g_{m}(k) \equiv \frac{k I_{m}^{\prime}(k)}{I_{m}(k)} \equiv m+\frac{k I_{m+1}(k)}{I_{m}(k)} .
\end{gathered}
$$

Решение уравнения (5) естественно искать в периодическом виде

$$
\alpha_{j}(t) \square \exp (i s t)
$$

где $s$ - частота капиллярных волн. Подставим (6) в (5) и получим дисперсионное уравнение задачи в виде

$$
s^{2} \mp \frac{2 \delta_{m}}{\beta_{m}} s-\frac{\kappa_{m}}{\beta_{m}}=0 .
$$

Напомним, что решается задача о пространственной неустойчивости струи. Но дисперсионное уравнение (7) имеет точно такой же аналитический вид, как в [17] для временной неустойчивости, хотя смысл частоты и волнового числа в этих двух постановках различен. Решения дисперсионного уравнения, полученные в [17], будем сравнивать с текущими результатами расчетов по (7). 

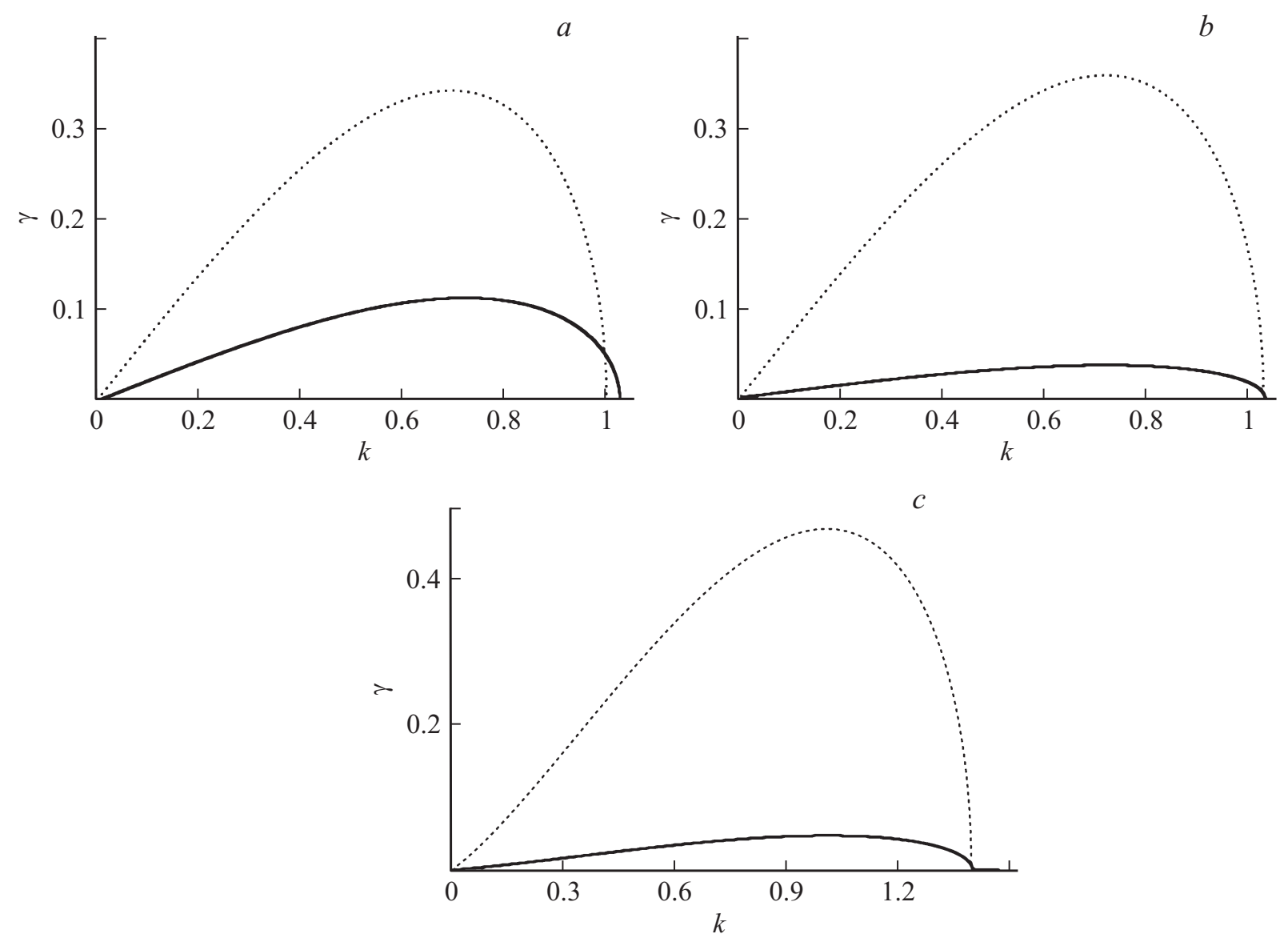

Рис. 1. Зависимость безразмерных инкрементов неустойчивости осесимметричных $(m=0)$ волн $\gamma_{k}$ (сплошная линия) и $\gamma_{s}$ (пунктирная линия) от вещественной части безразмерного волнового числа при $\rho=0.001 . a-w=0$ и $\mathrm{We}=0.01 ; b-w=0$ и $\mathrm{We}=0.1 ; c-w=1$ и $\mathrm{We}=0.1$.

Для исследования же пространственной неустойчивости струи, положим, что частота $s$ является вещественной, а волновое число $k-$ комплексным, и решим дисперсионное уравнение (7) относительно волнового числа $k$. Как отмечалось ранее, решение дисперсионного уравнения (7) относительно волнового числа в аналитическом виде невозможно, и решение (7) искалось в численном виде.

\section{Анализ результатов}

На рис. 1, $a$ представлена зависимость инкремента неустойчивости $\gamma_{k}=\operatorname{Im}(k)$ (сплошная линия), полученного в результате численного решения дисперсионного уравнения (7) относительно волнового числа $k$, и инкремента неустойчивости $\gamma_{s}=\operatorname{Im}(s)$ (пунктирная линия), полученного при решении дисперсионного уравнения (7) относительно частоты $s$ в [17], от вещественной части волнового числа. Как видно из рисунка, инкременты неустойчивости $\gamma_{k}$ и $\gamma_{s}$ расходятся не только по величине, но и по области определения, которая также является диапазоном неустойчивости волн. При детальном рассмотрении становится видно, что не совпада- ют длины наиболее неустойчивых волн, соответствующих максимальным значениям инкрементов. Однако при увеличении параметра We расхождение областей неустойчивости уменьшается, и при значении $\mathrm{We}=0.1$ (рис. $1, b, c$ ) исчезает не зависимо от величины заряда, приходящегося на единицу длины струи. Как показывают расчеты (см. рис. $1, a-c$ ) с увеличением параметров We и $w$ имеет место незначительное расширение области определения $\gamma_{k}$ и $\gamma_{s}$.

Аналогичная ситуация с длинами наиболее неустойчивых волн. При увеличении параметра We расхождение длин наиболее неустойчивых волн, полученных при исследовании пространственной и временной неустойчивостей, уменьшается. Так, при $\mathrm{We}=0.01$ волновое число наиболее неустойчивой волны, полученное при анализе пространственной неустойчивости, составляет $k_{\max }\left(\gamma_{k}\right)=0.726$, а волновое число наиболее неустойчивой волны, полученное при анализе временной неустойчивости, составляет $k_{\max }\left(\gamma_{s}\right)=0.6995$. При $\mathrm{We}=0.1$ их значения составят $k_{\max }\left(\gamma_{k}\right)=0.730$ и $k_{\max }\left(\gamma_{s}\right)=0.723$, а при $\mathrm{We}=0.3-k_{\max }\left(\gamma_{k}\right)=0.785$ и $k_{\max }\left(\gamma_{s}\right)=0.779$.

Так как исследования пространственной и временной неустойчивостей являются двумя разными математиче- 
скими подходами к исследованию устойчивости струи, то при схожих условиях должны одинаково описывать поведение системы.

Общий вид возмущения на поверхности струи можно записать в виде

$$
\xi \sim \alpha \exp [i s t+i k z+i m \varphi] .
$$

При исследовании временной неустойчивости волновое число считается вещественным, а частота является комплексной величиной, причем мнимая часть частоты определяет инкремент неустойчивости $\gamma_{s}$ :

$$
\begin{gathered}
\exp [i s t+i k z+i m \varphi]=\exp \left[i\left(\omega-i \gamma_{s}\right) t+i k z+i m \varphi\right] \\
=\exp \left[\gamma_{s} t\right] \exp [i \omega t+i k z+i m \varphi] .
\end{gathered}
$$

При исследовании пространственной неустойчивости частота считается вещественной $(s \equiv \omega)$, а волновое число - комплексным, и мнимая часть волнового числа определяет инкремент неустойчивости $\gamma_{k}$ :

$$
\begin{gathered}
\exp [i s t+i k z+i m \varphi]=\exp \left[i \omega t+i\left(k-i \gamma_{k}\right) z+i m \varphi\right] \\
=\exp \left[\gamma_{k} z\right] \exp [i \omega t+i k z+i m \varphi] .
\end{gathered}
$$

Так как оба подхода должны описывать одну и ту же волну при одинаковых внешних условиях, то

$$
\begin{gathered}
\exp \left[\gamma_{s} t\right] \exp [i \omega t+i k z+i m \varphi] \\
=\exp \left[\gamma_{k} z\right] \exp [i \omega t+i k z+i m \varphi], \\
\exp \left[\gamma_{s} t\right]=\exp \left[\gamma_{k} z\right], \\
\gamma_{s} t=\gamma_{k} z, \quad \frac{\gamma_{s}}{\gamma_{k}}=\frac{z}{t}=U_{0} .
\end{gathered}
$$

Таким образом, инкременты неустойчивости линейно связаны между собой, а коэффициентом пропорциональности является скорость движения струи относительно сопла или относительно внешней среды (в случае покоящейся внешней среды).

Действительно, для рис. $1, a$ скорость относительного движения струи и внешней среды составит $U_{0}=\sqrt{\mathrm{We} / \rho} \approx 3.16$ (напомним, что все величины безразмерны). Тогда величины инкрементов наиболее неустойчивых волн будут равны $\gamma_{k} \approx 0.115$ и $\gamma_{s} \approx 0.345$. Как видно, соотношение $\gamma_{s}=U_{0} \cdot \gamma_{k}$ выполняется в пределах погрешности численных вычислений. Так, для рис. $1, b U_{0} \approx 10$, а $\gamma_{k} \approx 0.036$ и $\gamma_{s} \approx 0.358$.

Расхождение областей реализации пространственной и временной неустойчивостей в области малых значений параметра We можно объяснить выходом за границы области применения модели короткой струи. Как правило, все реальные струи являются короткими (конечной длины) и движущимися, причем движение является неотьемлемой частью коротких струй. Весьма сложно представить себе покоящуюся короткую струю, также сложно наблюдать ее на практике, однако в отсутствие скорости движения струи рассмотрение сведется к исследованию устойчивости неподвижного цилиндрического столба жидкости. Так как цилиндрическая поверхность не обладает минимумом поверхностной энергии, она в конечном итоге распадется на сферические капли, в этом случае рассмотрение сведется к устойчивости поверхности неподвижной капли или капли, висящей на торце сопла, что не относится к рассматриваемой проблеме. При исследовании коротких струй скорость движения струи относительно сопла не может быть равной нулю. Данное ограничение накладывается и на модель пространственной неустойчивости струи: обращение скорости в ноль приводит к неопределенности в соотношении (8). Для справедливого выполнения условия (8) как перехода от временной неустойчивости к пространственной скорость не может стремиться к нулю, т. е. должна быть ограниченной снизу.

Опираясь на рис. 1, $b$ будем считать, что при значении $\mathrm{We}=0.1$ расхождение областей неустойчивости пренебрежимо мало, тем самым положив, что данная модель пространственной неустойчивости справедлива в области $\mathrm{We} \geq 0.1$. Все дальнейшее рассмотрение будем проводить только в рамках установленных ограничений.

Как показывают численные расчеты, зависимость инкрементов неустойчивости $\gamma_{k}=\operatorname{Im}(k)$ и $\gamma_{s}=\operatorname{Im}(s)$ от вещественной части волнового числа для осесимметричных волн при различных значениях параметра $w$ имеют качественно сходный характер. С увеличением $w$ области определения пространственной и временной неустойчивостей увеличиваются.

Как было показано в [18], для бесконечно длинной струи изгибные волны $(m=1)$ в отсутствие заряда на поверхности струи претерпевают неустойчивость при значениях параметра We $>1$. На рис. $2, a, b$ представлены зависимости инкрементов неустойчивости $\gamma_{k}=\operatorname{Im}(k)$ (сплошная линия) и $\gamma_{s}=\operatorname{Im}(s)$ (пунктирная линия) от вещественной части волнового числа для изгибных волн при различных значениях параметра We. Как видно из рисунков, в отсутствие заряда на поверхности струи пространственная неустойчивость изгибных волн также реализуется при значениях параметра We $>1$. Как показывают расчеты и видно из рис. 2, $c$, при наличии заряда на поверхности струи реализация пространственной неустойчивости изгибных волн имеет место уже при как угодно малых значениях параметра We, что также согласуется с результатами работы [18], выполненной для бесконечно длинной струи. Таким образом, для изгибных волн величины инкрементов пространственной и временной неустойчивостей связаны между собой, а также совпадают области реализации неустойчивостей.

Реализация неустойчивости изгибно-деформационных волн $(m=2)$ характеризуется большими критическими значениями параметров We и $w$, а так же смещением области неустойчивости в сторону коротких волн и наличием области устойчивости в длинноволновом диапазоне. На рис. 3 представлены зависимости инкрементов неустойчивости $\gamma_{k}=\operatorname{Im}(k)$ (сплошная линия) и 

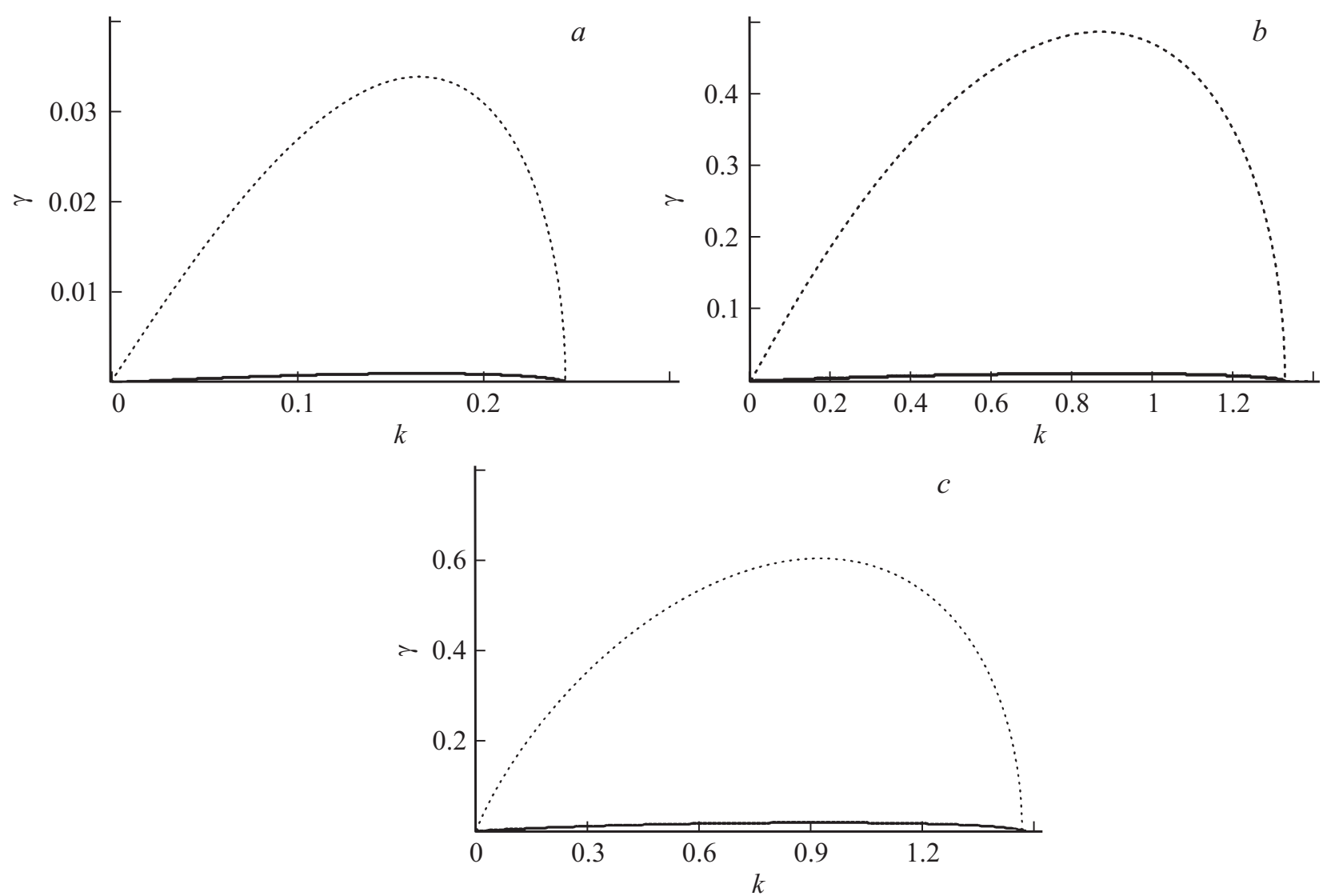

Рис. 2. Зависимость безразмерных инкрементов неустойчивости изгибных $(m=1)$ волн $\gamma_{k}$ (сплошная линия) и $\gamma_{s}$ (пунктирная линия) от вещественной части безразмерного волнового числа при $\rho=0.001 . a-w=0$ и $\mathrm{We}=1.1 ; b-w=0$ и $\mathrm{We}=2 ; c-$ $w=1$ и $\mathrm{We}=0.1$.

$\gamma_{s}=\operatorname{Im}(s)$ (пунктирная линия) от вещественной части волнового числа для изгибно-деформационных волн при различных значениях параметра We. Увеличение $w$ приводит к росту инкрементов и расширению диапазона волновых чисел, при которых неустойчивость имеет место. Как видно, и в этом случае величины инкрементов пространственной и временной неустойчивостей линейно связаны между собой.

Расчеты показывают, что при увеличении плотности внешней среды инкремент неустойчивости $\gamma_{k}$ увеличивается, в то время как изменения инкремента $\gamma_{s}$ незначительны. Однако соотношение между величинами инкрементов сохраняется.

\section{Оценки применимости модели}

Имея в виду короткую струю, вытекающую из металлического сопла, при наличии поля сил тяжести, действующих на струю при произвольной ее ориентации, целесообразно оценить применимость модели к условиям реального эксперимента.

В проведенных выше рассуждениях использовалась модель идеальной идеально электропроводной жидкости, но она может иметь отношение к реальным струям в ситуации, когда: вязкостью жидкости струи и среды можно пренебрегать; электропроводность жидкости достаточно высока, но электропроводность среды (которая, как правило, является газовой) достаточно мала. Выразим эти условия в форме условий на соответствующие безразмерные (чтобы не связываться с частностями) параметры

Что касается вязкости, то применимость модели определяется условием

$$
\mu \equiv v \sqrt{\rho_{1} / R \sigma} \ll 1
$$

Здесь $v-$ кинематическая вязкость жидкости; $\mu-$ ее безразмерная вязкость. При выполнении этого условия роль вязкости в реализации капиллярного волнового движения на поверхности струи незначительна, и жидкость можно считать идеальной. В этом соотношении обращает на себя внимание тот факт, что одну и ту же жидкость при фиксированной плотности жидкости и коэффициенте ее поверхностного натяжения можно считать и маловязкой и сильно вязкой в зависимости от размеров струи: толстые струи (больший радиус) будут маловязкими, а тонкие струи (маленький радиус) будут сильно вязкими. Так, в [24] было показано, что результаты расчетов величин инкрементов и волновых чисел 

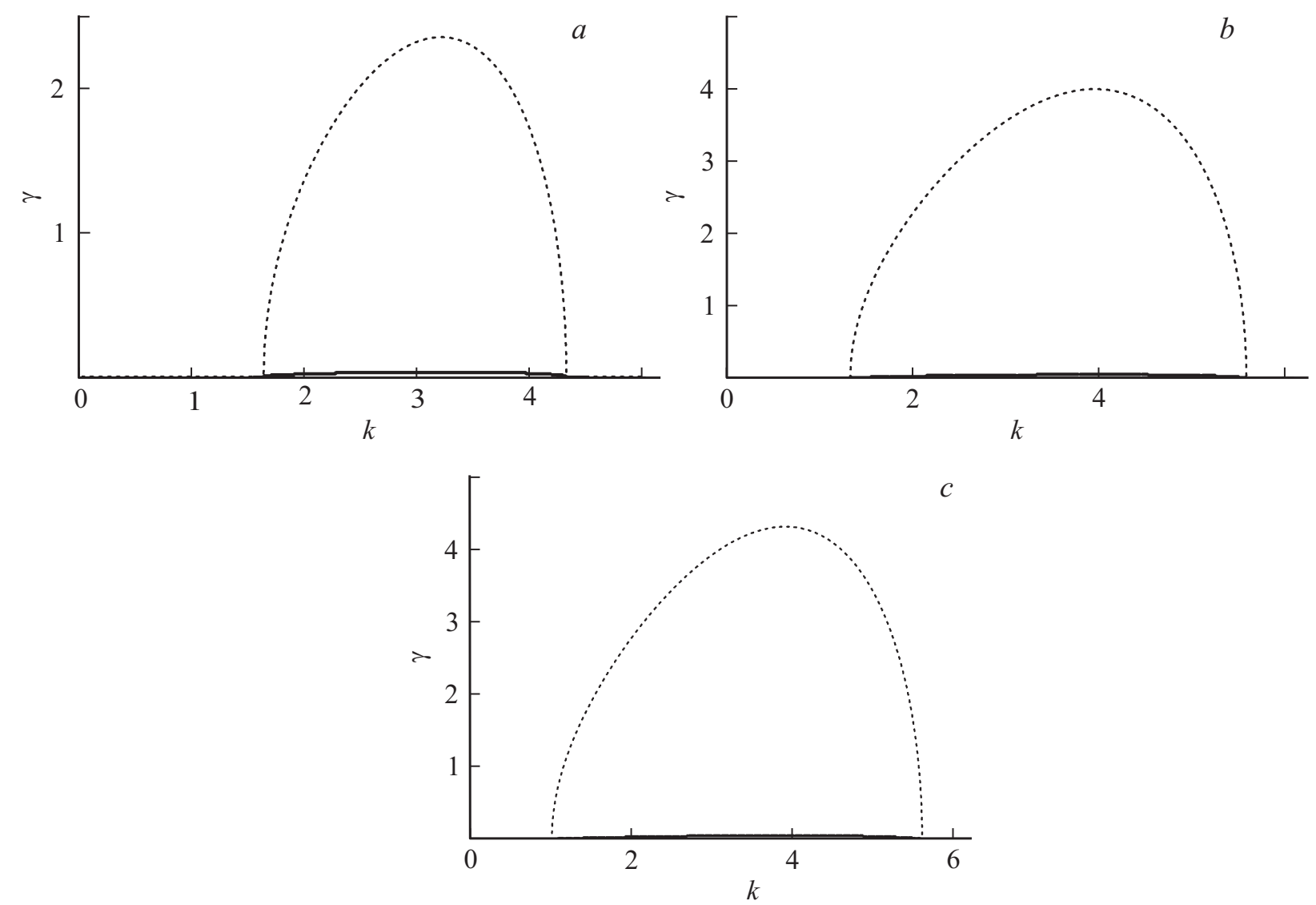

Рис. 3. Зависимость безразмерных инкрементов неустойчивости изгибно-деформационных $(m=2)$ волн $\gamma_{k}$ (сплошная линия) и $\gamma_{s}$ (пунктирная линия) от вещественной части безразмерного волнового числа при $\rho=0.001 . a-w=0$ и $\mathrm{We}=6$; $b-w=0$ и $\mathrm{We}=8 ; c-w=1$ и $\mathrm{We}=6$.

наиболее неустойчивых волн на поверхности струи для идеальной жидкости и жидкости с $\mu=0.1$ различаются не более чем на толщину линии. Увеличение $\mu$ на порядок приводит к существенной смене картины.

Что касается электропроводности жидкости, то ее можно считать идеально проводящей, если характерное максвелловское время (время выравнивания электрического потенциала) при волновом движении на поверхности струи много меньше характерного время отрыва капли, которое определяется наиболее неустойчивой модой.

Характерное время максвелловской релаксации для жидкости струи $\tau_{\varepsilon}$ определяется выражением

$$
\tau_{\varepsilon} \equiv\left(\varepsilon_{i n} / \eta_{i n}\right)
$$

где $\varepsilon_{i n}$ - диэлектрическая проницаемость жидкости, а $\eta_{\text {in }}$ - ее электропроводность.

Характерное время отрыва капли $\tau_{d}$, согласно [25], определяется выражением

$$
\tau_{d} \approx 8.46 \sqrt{\rho_{1} R^{3} / \sigma}
$$

Таким образом, условие применимости идеализации бесконечно большой электропроводности запишется в виде

$$
\tau_{\varepsilon \ll \tau_{d}}
$$

или

$$
\begin{aligned}
\varepsilon_{i n} / \eta_{i n} & \ll \frac{8.46 \sqrt{\rho_{1} R^{3}}}{\sqrt{\sigma}} \Rightarrow 1 / \eta_{\text {in }} \ll \frac{8.46 \sqrt{\rho_{1} R^{3}}}{\varepsilon_{i n} \sqrt{\sigma}} \\
& \Rightarrow \eta_{i n} \gg \frac{\varepsilon_{i n} \sqrt{\sigma}}{8.46 \sqrt{\rho_{1} R^{3}}} .
\end{aligned}
$$

Диэлектричность среды в условиях реального лабораторного эксперимента определится условием малости скорости утечки заряда с поверхности струи за счет проводимости среды по сравнению со скоростью поступления электрического заряда от металлического капилляра в жидкость. Это требование эквивалентно требованию, чтобы электропроводность среды, умноженная на длину не распавшейся части струи $L$, была много меньше электропроводности жидкости струи, умноженной на длину капилляра $l$, по которому жидкость подается в разрядную систему:

$$
\eta_{\text {in }} l \gg \eta_{e x} L
$$


Длина не распавшейся части струи, согласно [25] определяется выражением

$$
L \equiv U_{0} \tau_{d} \approx 8.46 U_{0} \sqrt{\rho_{1} R^{3} / \sigma}
$$

где $U_{0}-$ скорость движения струи. В итоге получим

$$
\eta_{i n} \gg \frac{8.46 U_{0} \sqrt{\rho_{1} R^{3}}}{l \sqrt{\sigma}} \eta_{e x} .
$$

В обсчитываемой модели дробления струй мы пренебрегаем влиянием поля тяжести. Это накладывает ограничение на применимость результатов расчетов к реальным процессам. Очевидно, что радиус струи должен быть в несколько раз меньше капиллярной постоянной жидкости. Для воды, например, при нормальной температуре капиллярная постоянная равна $0.22 \mathrm{~cm}$ [26].

Рассматривая роль поля тяжести в феномене выброса струй свободной поверхностью плоского мениска на торце капилляра, из которого вырывается струя в сильном (в два-три раза превышающем критическое значение для безграничной плоской поверхности) внешнем электростатическом поле, можно утверждать, что она ничтожна, и сам феномен не зависит от ориентации капилляра [27].

Чтобы влиянием силы тяжести на распад струи можно было пренебрегать, потребуем, чтобы за характерное время отрыва капли $\tau_{d}$ ее конец (на котором происходит отрыв капли) под действием силы тяжести сместился в направлении ее действия не более, чем на радиус струи. А именно потребуем, чтобы $g \tau_{d}^{2} / 2 \leq R$. Отсюда получим

$$
g \tau_{d}^{2} / 2 R \leq 1 \Rightarrow g \rho_{1} R^{2} / 2 \sigma \leq 1 .
$$

Такое условие выполнится для большинства струй, используемых в электродиспергировании $[21-23,28]$.

\section{Заключение}

Инкременты временной и пространственной неустойчивостей, характерных для идеализированных моделей бесконечно длинных и коротких струй, связаны между собой простым аналитическим выражением, которое проверено для осесимметричных $(m=0)$, изгибных $(m=1)$ и изгибно-деформационных $(m=2)$ капиллярных волн.

Численное исследование пространственной неустойчивости сопровождается большими затратами ресурсов и времени даже на современных компьютерах. Удобнее получить решения, описывающие бесконечные струи, в аналитическом виде и совершить переход к решениям, описывающим короткие струи.

Работа выполнена при поддержке грантов РФФИ 14-01-00170-а, 14-08-00240-а и 16-31-00095-мол-а/.

\section{Список литературы}

[1] Strutt J.W. (Lord Rayleigh) // Proc. of the London Math. Soc. 1878. Vol. 10. P. 4-13.

[2] Strutt J.W. (Lord Rayleigh) // Phil. Mag. 1892. Vol. 34. Ser. 5. P. $145-154$.

[3] Basset A.B. // Amer. J. Math. 1894. Vol. 16. P. 93-110.

[4] Weber C. // Z. Angew. Math. Mech. 1931. Bd. 11. H. 3. S. $136-154$.

[5] Strutt J.W. (Lord Rayleigh) // Phil. Mag. 1892. Vol. 34. Ser. 5. P. $177-180$.

[6] Taylor G., Van Dyke M.D. // Proc. Roy. Soc. London. 1969. Vol. 313A. P. 453-475.

[7] Betchov R., Criminale W.O. // Appl. Math. and Mechanics. 1967. Vol. 10. P. 332-362.

[8] Keller J.B., Rubinow S.I., Tu Y.O. // Phys. Fluids. 1973. Vol. 16. N 12. P. 2052-2055.

[9] Riahi D.N. // Appl. Mathematical Modelling. 2009. Vol. 33. P. 3546-3552

[10] Dressler J.L. // Phys. Fluids. 1998. Vol. 10. N 9. P. 2212-2227.

[11] Orizaga S, Riahi D.N., Hou S. // Int. J. Non-Linear Mechanics. 2014. Vol. 67. P. 218-230.

[12] Afzaal M.F., Uddin J., Alsharif A.M., Mohsin M. // Phys. Fluids. 2015. Vol. 27. P. 044106-1-12.

[13] Bogy D.B. // Ann. Rev. Fluid. Mech. 1979. Vol. 11. P. 207-228.

[14] Ентов В.М., Ярин А.Л. // ВИНИТИ. Итоги науки и техники. Сер. „Механика жидкости и газа“. 1984. Т. 17. С. $112-197$.

[15] Монодиспергирование вещества: принципы и применение // Е.В. Аметистов, В.В. Блаженков, А.К. Городов и др. / Под ред. В.А. Григорьева. М.: Энергоатомиздат, 1991. $336 \mathrm{c}$.

[16] Eggers J. // Rep. Prog. Phys. 2008. Vol. 71. N 036. P. 1-79.

[17] Shiryaeva S.O., Grigor'ev A.I. // Surf. Eng. Appl. Electrochem. 2014. Vol. 50. N 5. P. 395-404.

[18] Григорьев А.И., Ширяева С.О., Петрушов Н.А // ЖТФ. 2011. С. 81. Вып. 2. С. 16-22.

[19] Григорьев А., Петрушов Н., Ширяева С. // Изв. РАН. МЖГ. 2012. № 1. С. 68-79.

[20] Абрамовии М., Стиган И. Справочник по специальным функциям. М.: Наука, 1979. 830 с.

[21] Cloupeau M., Prunet Foch B. // J. Aerosol Sci. 1994. Vol. 25. N 6. P. $1021-1035$.

[22] Jaworek A., Krupa A. // J. Aerosol Sci. 1999. Vol. 30. N 7. P. 873-893.

[23] Kim O.V., Dunn P.F. // Langmuir. 2010. Vol. 26. P. 15807-15813.

[24] Ширяева С.О., Григорьев А.И., Левчук Т.В. // ЖТФ. 2003. т. 73. Вып. 11. С. 22-30.

[25] Левич В.Г. Физико-химическая гидродинамика. М.: Физматгиз, $1959.700 \mathrm{c}$.

[26] Ландау Л.Д., Лифиии, Е.М. Гидродинамика. М.: Наука, $1986.733 \mathrm{c}$.

[27] Григорьев А.И. // ЖТФ. 2007. Т. 77. Вып. 2. С. 31-40.

[28] Fenn J.B., Mann M. et al. // Science. 1989. Vol. 246. N 4926. P. 64-71. 